\title{
Monitoring of Thoracic Aortic Aneurysm in Blood by Fluorescence Spectroscopy
}

\author{
Zuzana Gul’ašová $^{1, *}$, Vladimíra Tomečková ${ }^{1}$, Miroslava Bilecová-Rabajdová ${ }^{1}$, Beáta Veliká ${ }^{1}$, Panayotis Artemiou ${ }^{2}$, \\ Vladimír Komanický, Mária Mareková ${ }^{1}$
}

\author{
${ }^{1}$ Department of Medical and Clinical Biochemistry, Pavol Jozef Šafárik University, Faculty of Medicine, Košice, Slovakia \\ ${ }^{2}$ Department of Cardiovascular Surgery, Pavol Jozef Šafárik University, Faculty of Medicine and VUSCH, Košice, Slovakia \\ ${ }^{3}$ Institute of Physics, Pavol Jozef Šafárik University, Faculty of Science, Košice, Slovakia \\ *Corresponding author: gulasova.z@gmail.com
}

Received May 24, 2015; Revised July 15, 2015; Accepted August 05, 2015

\begin{abstract}
Components of the blood plasma and serum represent a mixture of various endogenous substances with fluorescence properties. The aim of this work was a detection of pathological changes in blood of patients with thoracic aortic aneurysm by fluorescence spectroscopy and atomic force microscopy. The resulting autofluorescence of fluorophores in blood of patients with thoracic aortic aneurysm decreased in comparison with healthy subjects. The structure of the thoracic aorta was changed during the thoracic aortic aneurysm, what was manifested as the structural modifications in blood of patients observed by using atomic force microscopy. The fluorescence analysis and atomic force microscopy present new experimental ways in the study of the thoracic aortic aneurysm.
\end{abstract}

Keywords: thoracic aortic aneurysm, fluorescence spectroscopy, blood plasma and serum

Cite This Article: Zuzana Gulašová, Vladimíra Tomečková, Miroslava Bilecová-Rabajdová, Beáta Veliká, Panayotis Artemiou, Vladimír Komanický, and Mária Mareková, "Monitoring of Thoracic Aortic Aneurysm in Blood by Fluorescence Spectroscopy." American Journal of Medical and Biological Research, vol. 3, no. 5 (2015): 128-132. doi: 10.12691/ajmbr-3-5-2.

\section{Introduction}

Aneurysm of aorta is the most dangerous human cardiovascular disease which is characterized by 1.5 times enlargement of the aorta than normal size. The aneurysm is formed at the place where the vessel wall is weakened or has an abnormal structure. The thoracic aortic aneurysm, affecting a thoracic aorta, represents $25 \%$ of all cases of the aneurysm [4].

The aortic wall consists of three layers - tunica intima, tunica media and tunica adventitia. Each of these layers has a characteristic structure. There are elastic and collagen fibers [3], fibroblasts, vascular smooth muscle cells, specific glycoproteins, glykosaminoglycans and proteoglycans [15]. The basic structural and functional unit in the aortic wall is the lamellar unit. Each lamellar unit is composed of the vascular smooth muscle cells sandwiched between two layers of elastin fibers, which contain microfibrils and proteoglycans that form the extracellular matrix [5]. Lamellar units are intercalated by collagen fibers. Changes in the aortic wall during the aortic aneurysm include damage and fragmentation of elastin and collagen fibers, inflammatory process, failure of a secretory function of vascular smooth muscle cells and reduce their content in the aortic wall [2,15].

Aortic aneurysm is characterized by chronic inflammation and degradation of the extracellular matrix [19]. Molecular markers of the inflammatory process in the aortic aneurysm tissue are lipoxygenase, CRP-protein, interleukins, which impact on various components of the aortic wall [1]. Inflammation changes the metabolic processes occurring in the aortic tissue, tissue remodeling and the level of components in a patient blood. Macrophages located in the tunica adventitia product leukotriene D4 during the inflammatory process. The inflammatory mediator, leukotriene D4, stimulates the production of the macrophage inflammatory protein $1 \alpha$ (MIP1 $\alpha$ ) by macrophages, leading to recruitment of Tcells in the tunica adventitia. Leukotriene D4 (LTD4) also stimulates production of the macrophage inflammatory protein 2 (MIP2) by endothelial cells in the vasa vasorum, which leads to leukocyte recruitment in the tunica adventitia. These processes lead to release proteases such as matrix metalloproteinase-2 (MMP2), which cause matrix degradation [9].

Proteolysis of the extracellular matrix is associated with matrix metalloproteinases, mainly matrix metalloproteinase- 2 and matrix metalloproteinase-9. Matrix metalloproteinases constitute a group of the proteolytic enzymes containing a metallic ion $\left(\mathrm{Zn}^{2+}\right)$ in their active site. The proteolytic effects of MMPs play an important role in vascular remodeling, cellular migration and the processing of ECM proteins and adhesion molecules [6,24]. Matrix metalloproteinases can be produced by many cell types, including inflammatory cells (neutrophils and macrophages), vascular smooth muscle cells, also during matrix degradation and in response to inflammation and oxidation stresses [11,20]. Increased MMP activity has 
been reported in various inflammatory and degenerative disorders [2,21]. Loss of control of MMP activity can result in pathological vascular remodeling and vascular disease.

Matrix metalloproteinase-2 and matrix metalloproteinase-9 play a key role in thoracic aortic aneurysm. MMP-2 (gelatinase A) is expressed by smooth muscle cells and fibroblasts and also displays interstitial collagenase capabilities, cleaving Type I collagen [25]. MMP-9 (gelatinase B) may be produced by inflammatory cells such as neutrophils and macrophages [17]. Both enzymes are found especially in the tunica media, where contribute to the degradation of elastin and collagen fibers. Increased expression of MMP-2 and MMP-9 is typically during thoracic aortic aneurysm [13].

Transforming growth factor $\beta 1$ (TGF- $\beta 1$ ) is a key molecule in pathogenesis of thoracic aortic aneurysm. It has a central role in maintaining the extracellular matrix homeostasis [8], increased activity of TGF- $\beta 1$ leads to matrix degradation through increased production of plasminogen activators and release of matrix metalloproteinases-2 and 9 [11].

The aim of this work was a detection of pathological changes in blood in patients with thoracic aortic aneurysm by fluorescence spectroscopy and atomic force microscopy.

\section{Materials and Methods}

\subsection{Preparation of Samples}

Control group consisted of 20 healthy individuals. Experimental group comprised of 20 patients with the thoracic aortic aneurysm which was diagnosed clinically. Healthy individuals and patients were informed by their doctor about the aim of our experimental study. They were asked for consent to use their blood samples for our analysis. The samples of blood plasma and serum of patients and healthy individuals were centrifuged for 3 minutes at $3500 \mathrm{rpm}$ (BOECO centrifuge U-32R, Hamburg, Germany), and were kept in freezer at temperature $\mathrm{t}=-71^{\circ} \mathrm{C}$ (New Brunswick Scientific, Premium U410 Ultra-Low Temperature Freezer, Enfield, Connecticut, USA).

\subsection{Fluorescence Spectroscopy}

The phosphate buffer buffer $(0.2 \mathrm{M}$; $\mathrm{pH}=7.4)$ was prepared from $\mathrm{KH}_{2} \mathrm{PO}_{4}, \mathrm{Na}_{2} \mathrm{HPO}_{4}$ obtained from SigmaAldrich Chemie (Steinheim, Germany) and deionized water. The control and experimental blood plasma and serum samples diluted in the phosphate buffer $(0.2 \mathrm{M}$; $\mathrm{pH}$ $=7.4)$ in ratio (1:5000) were analysed using synchronous fluorescence fingerprints on Luminescence spectrophotometer Perkin Elmer LS 55 (Waltham, Massachusetts, USA) at room temperature $25^{\circ} \mathrm{C}$. The fluorescence measurements were performed at excitation wavelength from 200 to 400 $\mathrm{nm}$. Individual measurements were processed into contour and three-dimensional synchronous fluorescence fingerprints SFF using the software WinLab (Perkin Elmer, Waltham, Massachusetts, USA).

Synchronous excitation spectra of blood plasma and serum samples were the result of horizontal cut of SFF at $\Delta \lambda=50 \mathrm{~nm}$. The values of fluorescence intensity of control and experimental samples were statistically compared using Student - Newman - Keuls Multiple Comparisons Test.

\subsection{Atomic Force Microscopy}

Samples of blood plasma $(5 \mu l)$ and serum $(5 \mu l)$ layers deposited on the glass slides of patients and control group were analysed using an atomic force microscope Dimension Icon ${ }^{\circledR}$ (ICON, Bruker, Berkley, California, USA) in tapping mode with silicon tips (Mikro Masch, Berkley, California, USA, NSC35 series) with radius of curvature $\sim 10 \mathrm{~nm}$. The surface of each sample was processed by the software Scan Asyst ${ }^{\mathrm{TM}}$ and dried at room temperature.

\section{Results and Discussion}

Fluorescence spectroscopy is a method for for detection and determination the amount of fluorophores in the sample. Fluorophores in blood are organic substances which are capable to emit light after the adoption of energy [14]. On the resulting blood autofluorescence may contribute mainly characteristic proteins and coenzymes but also other substances which are different in patients compared to healthy persons. Several previously published papers described the use of fluorescence analytic method in the study of blood and tissue of patients with various diagnoses. The fluorescence analysis studied blood and urine of patients with cervical cancer [18], blood of patients with various stage of breast cancer [10] and blood of patients with limb ischemia [23]. Endogenous fluorophores of the aortic wall include proteins such as collagen and elastin which occur naturally in its structure.

One of the most important blood components is hemoglobin, which can exist in two forms as oxyhemoglobin and deoxyhemoglobin. The one peak has in $\lambda_{\mathrm{ex}}=555 \mathrm{~nm}$ deoxyhemoglobin, and oxyhemoglobin is characterized by two peaks at $\lambda_{\mathrm{ex}}=540,577 \mathrm{~nm}$ [27]. Another fluorophore - bilirubin, a product of hem catabolism, absorbs radiation and has a characteristic fluorescent excitation maximum at $\lambda_{\mathrm{ex}}=450 \mathrm{~nm}$.

Serum albumin, one of the most abundant proteins in the blood plasma and serum, plays an important role in the transport of a variety of endogenous and exogenous substances such as fatty acids, amino acids, hormones, vitamins [22], but it is also involved in maintaining the oncotic pressure. The albumin belongs to the endogenous fluorophores with fluorescent excitation maximum at $\lambda_{\text {ex }}=$ $280 \mathrm{~nm}$ and emission maximum at $\lambda_{\text {em }}=327 \mathrm{~nm}$ [26].

The important proteins of the blood include also globulins and a fibrinogen with the fluorescent excitation maximum $\lambda_{\mathrm{ex}}=280 \mathrm{~nm}$ and emission maximum $\lambda_{\mathrm{em}}=350 \mathrm{~nm}$ [7], which is not in the blood serum. During the blood coagulation the fibrinogen converts to fibrin, which is formed into fibrin fibers [12].

Three-dimensional synchronous fluorescent fingerprints of blood plasma of patients and healthy subjects are shown in Figure 1. This figure displays one fluorescence centrum located at $\Delta \lambda=70 \mathrm{~nm} / 278 \mathrm{~nm}$, but with a different fluorescent intensity $(F=373)$ in patients (Figure 1B) and healthy (Figure $1 \mathrm{~A})$ subjects $(\mathrm{F}=813)$. 


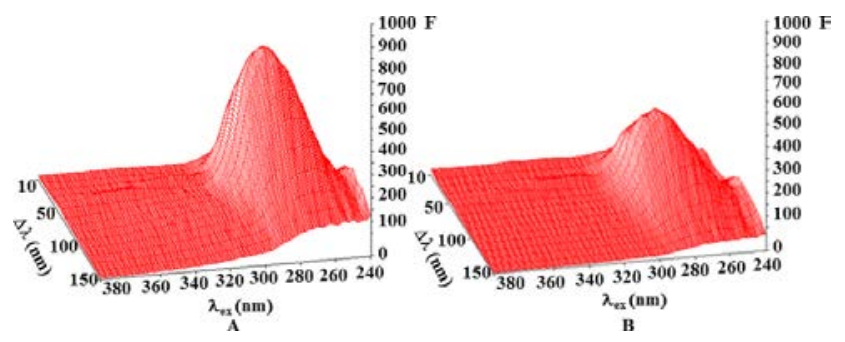

Figure 1. Circular characteristic pattern of three dimensional synchronous fingerprint of blood plasma of (A) healthy subjects and (B) patients with thoracic aortic aneurysm is result of fluorescence with the maximum fluorescence at $\Delta \lambda=70 \mathrm{~nm} / 278 \mathrm{~nm}$, showed a different fluorescent intensity $(\mathrm{F}=373)$ in patients with thoracic aortic aneurysm (B) and healthy (A) subjects $(\mathrm{F}=813) . \Delta \lambda=$ constant difference between emission and excitation synchronous spectra, $\lambda_{\mathrm{ex}}=$ excitation wavelength, $\mathrm{F}=$ fluorescence

Simple synchronous spectra (Figure 2) with maximum located at an excitation wavelength $\lambda_{\mathrm{ex}}=280 \mathrm{~nm}$ showed a significant decrease of the fluorescence intensity $(F=373)$ of blood plasma of patients (Figure 2B) compared to control (Figure 2A) group $(\mathrm{F}=831)$. At an excitation wavelength $\lambda_{\mathrm{ex}}=360 \mathrm{~nm}$ we did not notice a significant difference between patients $(\mathrm{F}=26)$ and control group $(F=32)$. The fluorescent maximum, which is located at $\lambda_{\text {max }}=350-360 \mathrm{~nm}$, graphically presents a mixture various fluorophores for example $\mathrm{NADH}+\mathrm{H}^{+}$, proteins, porphyrins [14].
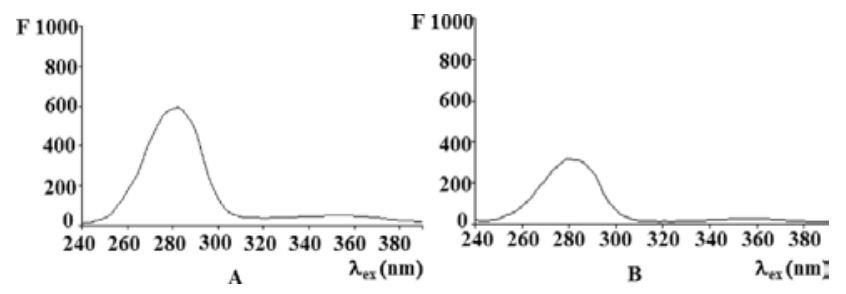

Figure 2. Horizontal section of synchronous fluorescence fingerprints $(\Delta \lambda=50 \mathrm{~nm})$ of blood plasma showed the significant decrease of fluorescent intensity in the group of patients with thoracic aortic aneurysm (B) at $\lambda_{\mathrm{ex}}=280 \mathrm{~nm}$ in comparison with healthy subjects (A). The fluorescent intensity $\lambda_{\mathrm{ex}}=280$ is typical for the proteins and aromatic amino acids. $\Delta \lambda=$ constant difference between emission and excitation synchronous spectra, $\lambda_{\mathrm{ex}}=$ excitation wavelength

Enzyme cofactors are also characterized by fluorescence properties. Reduced nicotinamide adenine dinucleotide $\left(\mathrm{NADH}+\mathrm{H}^{+}\right)$is a highly fluorescent compound which has an absorption maxim at $\lambda_{\mathrm{ex}}=340-$ $350 \mathrm{~nm}$ and an emission maxim at $\lambda_{\text {em }}=460 \mathrm{~nm}$. Oxidized flavine adenine dinucleotide (FAD ${ }^{+}$) absorbs a light at $\lambda_{\text {ex }}$ $=450 \mathrm{~nm}$ and emits at $\lambda_{\mathrm{em}}=525 \mathrm{~nm}$ [14]. If the cell has sufficient oxygen, the production of $\mathrm{NADH}+\mathrm{H}^{+}$decreases, thereby the fluorescence intensity decreases. If the cell has the absence of oxygen, the production of $\mathrm{NADH}+\mathrm{H}^{+}$ increases, which also leads to an increase in fluorescence. The presence of amino acids, reduced $\mathrm{NADH}+\mathrm{H}^{+}$, oxidized $\mathrm{FAD}^{+}$, porphyrins, collagen, elastin and other proteins of cells and tissues increase in the fluorescence. The fluorescence of proteins shows generally a structural arrangement of the cell and tissue. The presence of other fluorophores, such as pyridine and pyrrole derivatives, is a result of metabolic activities in the cell [14].

Fluorescence signals (at $\lambda_{\mathrm{ex}}=280 \mathrm{~nm}, 350 \mathrm{~nm}$ ) of blood plasma of patients with thoracic aortic aneurysm were compared statistically with healthy subjects (Figure 3). Results displayed a significant $(\mathrm{p}<0.001)$ reduction of autofluorescence in patients (Figure 3A) with thoracic aortic aneurysm at $\lambda_{\mathrm{ex}}=280 \mathrm{~nm}$, while a slight decrease of fluorescence signal (Figure 3B) was observed in patients at $\lambda_{\text {ex }}=350 \mathrm{~nm}$. The fluorescence signal at $\lambda_{\mathrm{ex}}=280 \mathrm{~nm}$ is typical for the aromatic amino acids (Phe, Tyr, Trp) and proteins (albumin, fibrinogen).

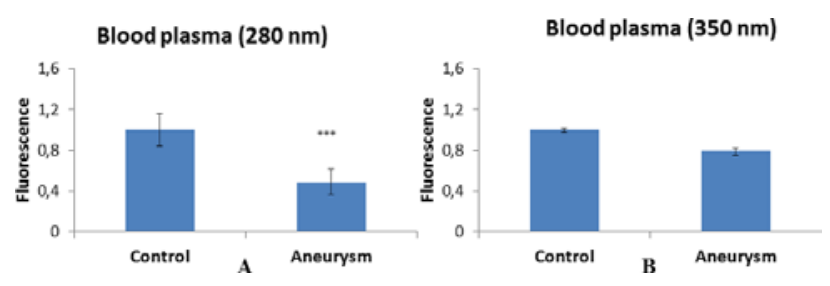

Figure 3. Fluorescence signals (at $\lambda_{\mathrm{ex}}=280 \mathrm{~nm}, 350 \mathrm{~nm}$ ) of blood plasma of patients with thoracic aortic aneurysm were compared statistically with healthy subjects. Results displayed a significant $(p<0.001)$ reduction of autofluorescence in patients with thoracic aortic aneurysm at $\lambda_{\mathrm{ex}}=280 \mathrm{~nm}$ (A), while a slight decrease of fluorescence signal was observed in patients at $\lambda_{\mathrm{ex}}=350 \mathrm{~nm}$ (B). $\lambda_{\mathrm{ex}}=$ excitation wavelength

The three-dimensional synchronous fluorescence fingerprint of patient blood serum (Figure 4B) exhibited lower fluorescence signal $(\mathrm{F}=332)$ in comparison with $(\mathrm{F}$ $=501$ ) of healthy subjects blood serum (Figure 4A) but showed a similar fluorescent center $(\Delta \lambda=70 \mathrm{~nm} / 277 \mathrm{~nm})$.

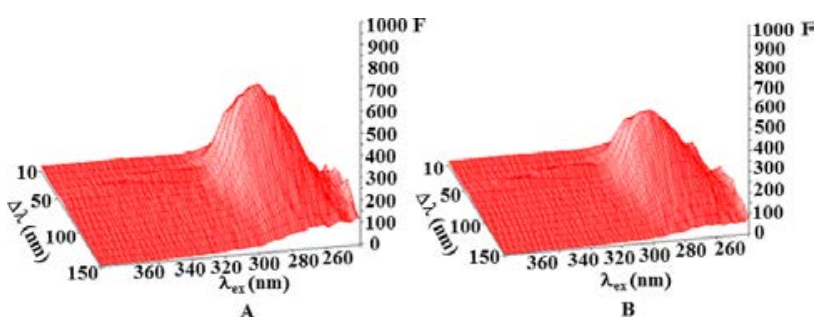

Figure 4. Circular characteristic pattern of three dimensional synchronous fingerprint of blood serum of (A) healthy subjects and (B) patients with thoracic aortic aneurysm is result of fluorescence with the maximum fluorescence at $\Delta \lambda=70 \mathrm{~nm} / 277 \mathrm{~nm}$. The three-dimensional synchronous fluorescence fingerprint of blood serum of patients (B) exhibited lower fluorescence signal $(\mathrm{F}=332)$ in comparison with $(\mathrm{F}=$ 501) blood serum of healthy subjects (A). $\Delta \lambda=$ constant difference between emission and excitation synchronous spectra, $\lambda_{\mathrm{ex}}=$ excitation wavelength, $\mathrm{F}$ = fluorescence

The decrease of fluorescence signal $(F=308)$ of the blood serum of the patients (Figure 5B) with thoracic aortic aneurysm in comparison with healthy subjects ( $\mathrm{F}=$ 480 , Figure $5 \mathrm{~A}$ ) was detected at the simple synchronous spectra (at $\lambda_{\mathrm{ex}}=280 \mathrm{~nm}$ ) where emit the maximum fluorescence signal the mixture of proteins and aromatic amino acids. The comparison of fluorescence signal (at $\lambda_{\text {ex }}$ $=350 \mathrm{~nm}$ ) of blood serum of patients with thoracic aortic aneurysm $(F=34)$ with healthy subjects $(F=36)$ showed similar the fluorescence signal (Figure 5B).

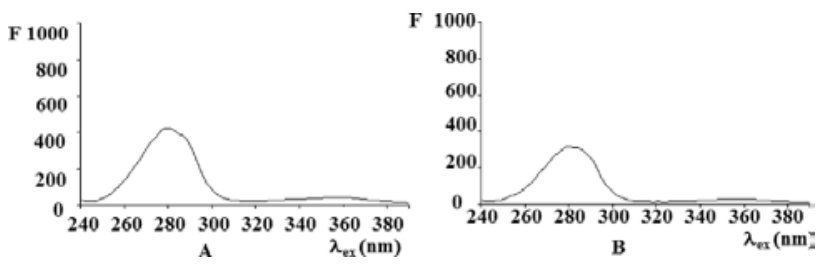

Figure 5. Horizontal section of synchronous fluorescence fingerprints $(\Delta \lambda=50 \mathrm{~nm})$ of blood serum showed the significant decrease of fluorescent intensity in the group of patients with thoracic aortic aneurysm (B) at $\lambda_{\mathrm{ex}}=280 \mathrm{~nm}$ in comparison with healthy subjects (A). The fluorescent intensity $\lambda_{\text {ex }}=280$ is typical for the proteins and aromatic amino acids. $\Delta \lambda=$ constant difference between emission and excitation synchronous spectra, $\lambda_{\mathrm{ex}}=$ excitation wavelength 
Composition of blood serum is a dynamic system. Proteins of blood serum play different biochemical roles in several metabolic processes in a body. Statistical comparison of fluorescence signal (at $\lambda_{\mathrm{ex}}=280 \mathrm{~nm}$ ) of blood serum of patients with thoracic aortic aneurysm and healthy subjects showed a significant $(p<0.01)$ reduction of the autofluorescence (Figure 6A) in comparison to control group. The fluorescence signal (at $\lambda_{\text {ex }}=350 \mathrm{~nm}$ ) of blood serum of patients with thoracic aortic aneurysm was compared statistically with healthy subjects and the results displayed a slight decrease of fluorescence signal (Figure 6B).
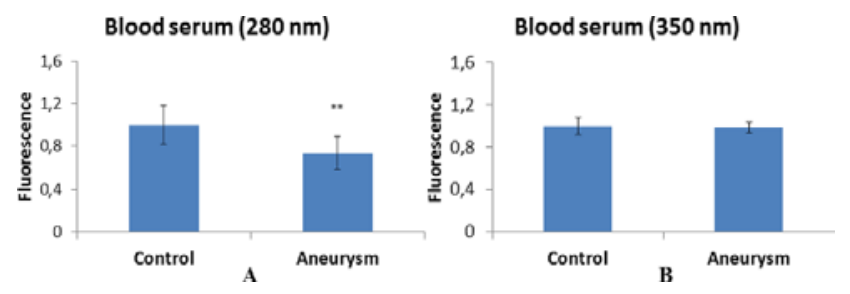

Figure 6. Fluorescence signals (at $\lambda_{\mathrm{ex}}=280 \mathrm{~nm}, 350 \mathrm{~nm}$ ) of blood serum of patients with thoracic aortic aneurysm were compared statistically with healthy subjects. The significant $(p<0.01)$ reduction of the autofluorescence was noticed in blood serum of patients with thoracic aortic aneurysm at $\lambda_{\mathrm{ex}}=280 \mathrm{~nm}$ in comparison with control group healthy subjects $(\mathrm{A})$, but at $\lambda_{\mathrm{ex}}=350 \mathrm{~nm}$ results displayed a slight decrease of fluorescence signal (B). $\lambda_{\mathrm{ex}}=$ excitation wavelength

Atomic force microscopy can display surface of fluid and solid materials [16] and it showed small $30 \mathrm{~nm}$ in blood plasma of healthy subjects (Figure 7A) in comparison with the well-defined circular $400 \mathrm{~nm}$ large globules in experimental samples (Figure 7B). This method detected higher roughness ( $\mathrm{rq}=9.24-12 \mathrm{~nm}$ ) of experimental samples of blood plasma (Figure 7B) in comparison with healthy subjects $(\mathrm{rq}=1.35 \mathrm{~nm})$.
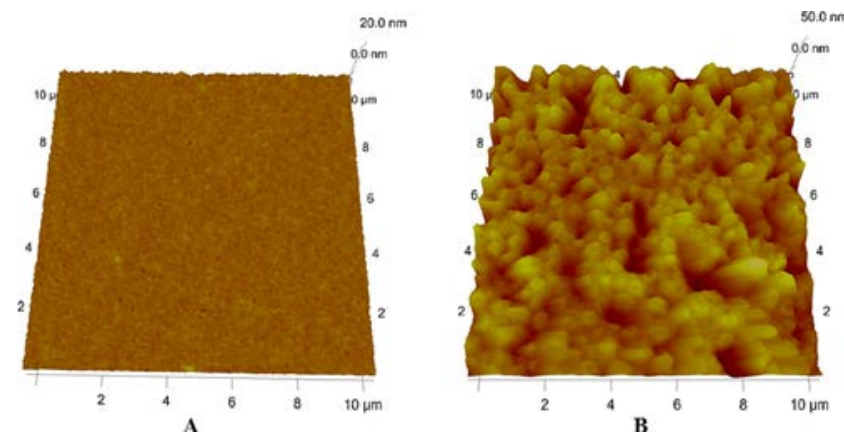

Figure 7. Atomic force microscopy shows the surface of blood plasma of (A) healthy subjects and (B) patients with thoracic aortic aneurysm. "0 nm” and "20 - 50nm” indicates ( $\mathrm{z}$ axis), minimal and maximal height of each globule
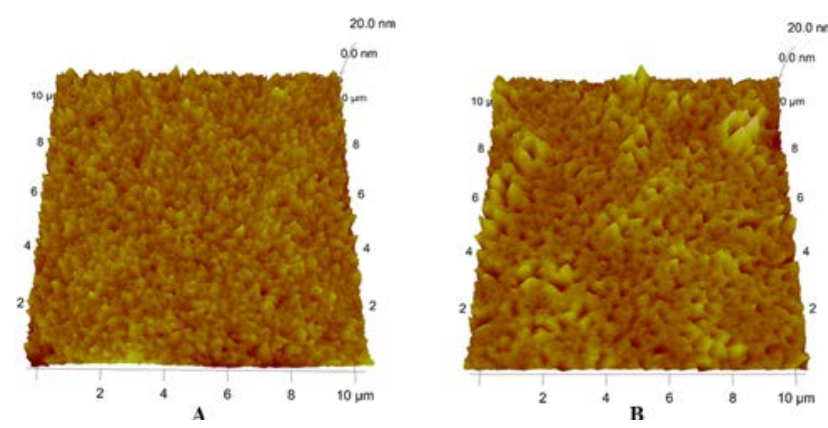

Figure 8. Atomic force microscopy shows the surface of blood serum of healthy subjects (A) and patients with thoracic aortic aneurysm (B). "0 $\mathrm{nm}$ ” and "20 nm” indicates ( $\mathrm{z}$ axis), minimal and maximal height of each globule
The higher roughness $(2.55-2.74 \mathrm{~nm})$ of experimental samples in comparison with healthy subjects $(2.06 \mathrm{~nm})$ was observed also in blood serum (Figure 8A). Atomic force microscopy revealed in blood serum of patients with thoracic aortic aneurysm several irregular various globules (50 - $100 \mathrm{~nm}$ ) holes and globules which merge into 700 $\mathrm{nm}$ larger structures (Figure 8B). The roughness of these samples was similar to the roughness of the control sample of the blood serum where occurred of the size (50 $-100 \mathrm{~nm})$.

\section{Conclusion}

The degradation and remodelling of the extracellular matrix of aortic tissue lead to inflammation and fragmentation of structural components in the aortic wall. The characterization of metabolic and pathological changes in cells and tissues of patients with thoracic aortic aneurysm were investigated by synchronous fluorescence fingerprint, a sensitive method which was able to detect the decrease of fluorophores in blood of patients. The modified structure of blood was revealed also by atomic force microscopy in the blood of patients with thoracic aortic aneurysm. These experimental methods could contribute to the improvement of diagnosis of the cardiovascular disease affecting the thoracic aorta in the future.

\section{Acknowledgement}

This work was was supported by the Slovak Academy of Science and the Slovak Grant Agency for Science under the contract No. VEGA-1/0115/14.

\section{List of Abbreviations}

$\mathrm{F}=$ fluorescence, $\mathrm{FAD}^{+}=$flavin adenine dinucleotide, LTD4 = leukotriene D4, MIP1 $\alpha=$ macrophage inflammatory protein $1 \alpha$, MIP2 = macrophage inflammatory protein 2 , MMPs = matrix metalloproteinases, MMP-2 = matrix metalloproteinase-2, MMP-9 = matrix metalloproteinase-9, $\mathrm{NADH}+\mathrm{H}^{+}=$reduced nicotinamide adenine dinucleotide, Phe $=$ phenylalanine, $\mathrm{rq}=$ roughness, $\mathrm{TGF}-\beta 1=$ transforming growth factor $\beta 1$, Trp = tryptophan, Tyr $=$ tyrosine, $\lambda_{\text {ex }}=$ excitation wavelength, $\Delta \lambda=$ constant difference between emission and excitation synchronous spectra.

\section{References}

[1] Artemiou, P., Charokopos, N., Rouska, E., Sabol, F., Chrysogonidis, I., Tsavdaridou, V., Paschalidis, G, "C-Reactive protein/interleukin-6 ratio as marker of the size of the uncomplicated thoracic aortic aneurysms", Interact Cardiovasc Thorac Surg. 15, 871-877, 2012.

[2] Barbour, J.R.., Spinale, F.G., Ikonomidis, J.S, "Proteinase systems and thoracic aortic aneurysm progression", J Surg Res, 139 (2), 292-307, 2006.

[3] Berillis, P, "The role of Collagen in the Aorta's Structure", The Open Circulation \& and Vascular Journal, 6, 1-8, 2013.

[4] Coady, M.A., Rizzo, J.A., Elefteriades, J.A, "Natural history, pathogenesis, and etiology of thoracic aortic aneurysms and dissections", Cardiol Clin, 17, 615-633, 1999. 
[5] El-Hamamsy, I., Yacoub, M.H, "Cellular and molecular mechanisms of thoracic aortic mechanisms", Nat Rev Cardiol, 6 (12), 771-786, 2012.

[6] Galis, Z.S., Khatri, J.J, "Matrix metalloproteinases in vascular remodelling and atherogenesis: The good, the bad, and the ugly", Circ Res, 90, 251-262, 2002.

[7] Goncalves, S., Santos, N.C., Martins-Silva, J., Saldanha, C, "Fibrinogen-beta-estradiol binding studied by fluorescence spectroscopy: denaturation and pH effects", J Fluoresc, 16, 207213, 2006.

[8] Goumans, M.J., Liu, Z., Ten Dijke, P, "TGF- $\beta$ signaling in vascular biology and dysfunction", Cell Res, 19, 116-127, 2009.

[9] He, R., Guo, D-Ch., Sun, W., Papke, Ch.L., Duraisamy, S., Estrera, A. L.; Safi, H.J.; Ahn, Ch.; Buja, L.M.; Arnett, F.C.; Zhang, J., Geng, Y.J.; Milewicz, D.M, "Characterization of the inflammatory cells in ascending thoracic aortic aneurysms in patients with Marfan syndrome, familial thoracic aortic aneurysms, and sporadic aneurysms", J Thorac Cardiovasc Surg, 136, 922-929, 2008.

[10] Kalaivani, R., Masilami, V., Sivaji, K., Elangovan, M., Selvaraj, V., Balamurugan, S.G., Al-Salhi, M.S, "Fluorescence Spectra of Blood Components for Breast Cancer Diagnosis", Photomed Laser Surg, 26, 251-256, 2008.

[11] Kim, E.S., Kim, M., Moon, A, "TGF- $\beta$-induced upregulation of MMP-2 and MMP-9 depends on p38 MAPK, but not ERK signaling in MCF-1OA human breast epithelial cells", Int J Oncol, 25, 1375-1382, 2004.

[12] Kollman, J.M., Pandi, L., Sawaya, M.R., Riley, M., Doolittle, R.F, "Crystal structure of human fibrinogen", Biochemistry, 48, 38773886, 2009.

[13] Koullias, G.J., Ravichandran, P., Korkolis, D.P., Rimm, D.L., Elefteriades, J.A, "Increased tissue microarray matrix metalloproteinase expression favors proteolysis in thoracic aortic aneurysms and dissections", Ann Thorac Surg, 78, 2106-2110, 2004.

[14] Lackowicz, J.R.: Principles of Fluorescence Spectroscopy. Third edition, Springer Science + Business Media, LLC, New York, USA, 2006, 63-66.

[15] Lacolley, P., Regnault, V., Nicoletti, A., Li, Z., Michel, J.-B, "The Vascular Smooth Muscle Cell in arterial pathology: a cell that can take on multiple roles", Cardiovasc Res, 95, 194-204, 2012.
[16] Lee, CH.K., Wang, Y.S., Huang, L.S., Lin, S, "Atomic force microscopy: Determination of unbiding force, off rate and energy barrier for protein-ligand interaction", Micron, 38, 446-461, 2007.

[17] Longo, G.M., Xiong, W., Greiner, T.C., Zhao, Y., Fiotti, N., Baxter, B.T, "Matrix metalloproteinases 2 and 9 work in concert to produce aortic aneurysms", J Clin Invest, 110, 625-632, 2002.

[18] Masilamani, V., Alsalhi, M.S., Vijmasi, T., Govindarajan, K., RathanRai, R., Atif, M., Prasad, S., Aldwayyan, A.S, "Fluorescence spectra of blood and urine for cervical cancer detection", J Biomed Opt, 17, 098001.

[19] Nagasawa, A., Yoshimura, K., Suzuki, R., Mikamo, A., Yamashita, O., Ikeda, Y., Tsuchida, M., Hamano, K, "Important role of the angiotensin II in producing matrix metalloproteinase-9 in human thoracic aortic aneurysms", J Surg Res, 183, 472-477, 2013.

[20] Račay, P., Chomová, M., Tatarková, Z., Kaplán, P., Hatok, J., Dobrota D, "Ischemia-induced mitochondrial apoptosis is significantly attenuated by ischemic preconditionic", Cell $\mathrm{Mol}$ Neurobiology, 29. 6-7, 901-908, 2009.

[21] Raffetto, J.D., Khalil, R.A, "Matrix metalloproteinases and their inhibitors in vascular remodeling and vascular disease", Biochem Pharmacol, 75, 346-359, 2008.

[22] Tan, M., Liang, W., Luo, X., Gu, Y, "Fluorescence Spectroscopy Study on the Interaction between Evodiamine and Bovine Serum Albumin", J Chem, 2013, 1-6, 2013.

[23] Tomečko, M., Sabol, F., Frankovičová, M., Petrášová, D, "Monitorovanie ischemickej choroby končatiny pomocou merania autofluorescencie krvi", Ateroskleróza, 3-4, 477-482, 2013.

[24] Visse, R., Nagase, H, "Matrix metalloproteinases and tissue inhibitors of metalloproteinases: Structure, function, and biochemistry", Circ Res, 92, 827-839, 2003.

[25] Zervoudaki, A., Economou, E., Stefanadis, C., Pitsavos, C., Tsioufis, K., Aggeli, C., Vasiliadou, K., Toutouza, M., Toutouzas, $\mathrm{P}$, "Plasma levels of active extracellular matrix metalloproteinases 2 and 9 in patients with essential hypertension before and after antihypertensive treatment", J Hum Hypertens, 17, 119-124, 2003.

[26] Zhang, G., Que, Q., Pan, J., Guo, J, "Study of the interaction between icariin and human serum albumin by fluorescence spectroscopy", J Mol Struct, 881, 132-138, 2008.

[27] Zheng, W., Dong, L., Zeng, Y., Luo, Y., Qu, J.Y, "Two-photon excited hemoglobin fluorescence", Biomed Opt Express, 2, 71-79, 2011. 